\title{
DESIGN OF A LOW LOSS SRF CAVITY FOR THE ILC
}

\author{
J. Sekutowicz, DESY, 22607 Hamburg, Germany \\ K. Ko, L. Ge, L. Lee, Z. Li, C. Ng, G. Schussman, L. Xiao, SLAC, Menlo Park, 94025 California, USA \\ I. Gonin, T. Khabibouline, N. Solyak, FNAL, Batavia, 60510-0500 Illinois, USA \\ Y. Morozumi, K. Saito, KEK, Tsukuba, 305-0801, Japan \\ P. Kneisel, TJNAF (JLAB), Newport News, 23606 Virginia, USA
}

\begin{abstract}
An international team comprising SLAC, KEK, FNAL, JLAB and DESY is collaborating on the design, fabrication and test of a low loss, $1.3 \mathrm{GHz}$ 9-cell SRF structure as a potential improvement for the ILC main linac. The advantages of this structure over the TESLA structure include lower cryogenic loss, shorter rise time, and less stored energy. Among the issues to be addressed in this design are HOM damping, Lorentz force detuning and multipacting. We will report on HOM damping calculations using the parallel finite element eigenmode solver Omega3P and the progress made towards an optimized design. Studies on multipacting and estimates of the Lorentz force detuning will also be presented.
\end{abstract}

\section{INTRODUCTION}

At the beginning of the TESLA Collaboration, in 1992, the shape of the TESLA cavity was proposed [1]. The cavity was optimized mainly with respect to $E_{\text {peak }} / E_{\text {acc }}$, the ratio of maximum electric field on the metal wall to the accelerating gradient. The low value of $\mathrm{E}_{\text {peak }} / \mathrm{E}_{\mathrm{acc}}=2$ was, and still is, very helpful in suppressing electron field emission at high gradients when the surface cleaning and final assembly, playing still the major role, are done in a proper way. Since that time remarkable progress in these procedures had been made and many single-cell and several multi-cell cavities were tested at very high gradients $(>35 \mathrm{MV} / \mathrm{m})$, showing no field emission from residual particulates on the wall. After more than 12 years one can review the arguments and ask the question again: what is the optimum shape that allows maximum gradients limited this time by the physical property of the bulk niobium, namely maximum magnetic flux $\mathrm{B}_{\text {peak }}$ on the metal wall? The critical flux $\mathrm{B}_{\mathrm{c}}$ is app. $180 \mathrm{mT}$ at which niobium becomes normal conducting. The geometry optimization would be then aimed towards a lower $\mathrm{B}_{\text {peak }} / \mathrm{E}_{\text {acc }}$ ratio that can be achieved by closing the irises and increasing the cavity volume where the magnetic energy is stored. In addition, this way of optimization increases the $(\mathrm{R} / \mathrm{Q})$ value and the geometric factor $\mathrm{G}$ making the cavity more effective in acceleration because of higher gradients and less energy dissipation for the same stored energy. The measure of the effectiveness is the product (R/Q).G. The "price" for all afore-mentioned improvements is an enhancement of $\mathrm{E}_{\text {peak }} / \mathrm{E}_{\text {acc }}$ ratio and the increased loss factors for monopole and dipole parasitic modes. Also the cell-to-cell coupling $\mathrm{k}_{\mathrm{cc}}$ decreases while the field flatness of the fundamental mode becomes more sensitive to frequency errors of the individual cells. The figure of merit for the sensitivity, a fast increase with the number of cells $\mathrm{N}_{\mathrm{c}}$ in a multi-cell structure, is the factor: $\left(\mathrm{N}_{\mathrm{c}}\right)^{2} / \mathrm{k}_{\mathrm{cc}}$. Fortunately we observe here also an improvement. Many TESLA 9-cell structures built for the TTF linac had been pre-tuned and after the final chemical treatment and cleaning their field flatness was well preserved.

Taking all these advantages and disadvantages into account the Low Loss cavity shape with high value of (R/Q) $\cdot \mathrm{G}$ was proposed for the CEBAF $12 \mathrm{GeV}$ upgrade in 2002 [2]. For this case, the low beam current $(<0.5 \mathrm{~mA})$, small number of cells in the structure (only 7$)$, and moderate operating gradient (19 MV/m), compensate for all the drawbacks of this cavity design. The main advantage of the LL shape is potentially low cryogenic loss that will allow the operation of 80 upgrade cavities in addition to the 320 cavities presently in use, all within the capability of the existing Jlab cryoplant.

In 2004 it was proposed [3, 4] to adapt the $1.5 \mathrm{GHz}$ LL CEBAF shape to $1.3 \mathrm{GHz}$ frequency of the fundamental mode (FM) and to check its possible advantages and disadvantages as the cavity shape for the main accelerator of the International Linear Collider project after the decision was made to proceed the effort with the superconducting technology. In the next sections we will present results of our work towards the goal of designing a LL cavity for the ILC.

\section{INNER CELL}

RF-parameters of the accelerating mode in a multi-cell structure are dominated by the shape of an inner cell. The original TESLA inner cell and the new ILC LL cell are shown in Fig. 1. Their RF-parameters are listed in Table 1. First, the new shape has Bpeak/Eacc lower by $10 \%$ therefore its ultimate gradient is $50 \mathrm{MV} / \mathrm{m}$. The cryogenic loss of this shape is also $23 \%$ lower then the TESLA shape for the same surface resistance at $1.3 \mathrm{GHz}$. The biggest drawback is the enhanced Epeak/Eacc ratio which together with smaller iris diameter and almost parallel cavity side

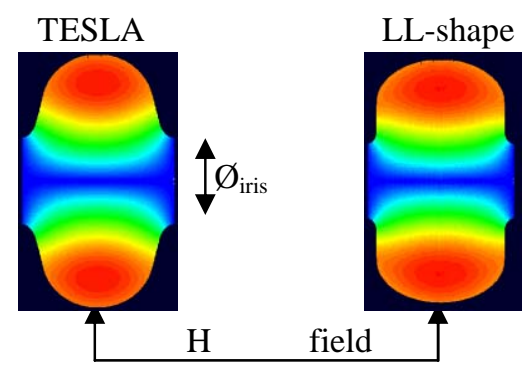

Figure 1: $\mathrm{H}$ contour in two shapes of inner cell. 
Table 1. RF parameters of both inner cells.

\begin{tabular}{|l|c|c|c|}
\hline \multicolumn{1}{|c|}{ Parameter } & Unit & TESLA & LL-Shape \\
\hline Øiris & {$[\mathrm{mm}]$} & 70 & 60 \\
\hline $\mathrm{k}_{\mathrm{cc}}$ & {$[\%]$} & 1.9 & 1.52 \\
\hline $\mathrm{E}_{\text {peak }} / \mathrm{E}_{\mathrm{acc}}$ & - & 1.98 & 2.36 \\
\hline $\mathrm{B}_{\text {peak }} / \mathrm{E}_{\text {acc }}$ & {$\left[\mathrm{mT} \cdot(\mathrm{MV} / \mathrm{m})^{-1}\right]$} & 4.15 & 3.61 \\
\hline Lorentz factor ${ }^{*}, \mathrm{k}_{\mathrm{L}}$ & {$\left[\mathrm{Hz} \cdot(\mathrm{MV} / \mathrm{m})^{-2}\right]$} & -0.74 & -0.81 \\
\hline $\mathrm{R} / \mathrm{Q}$ & {$[\Omega]$} & 113.8 & 133.7 \\
\hline $\mathrm{G}$ & {$[\Omega]$} & 271 & 284 \\
\hline $\mathrm{R} / \mathrm{Q} \cdot \mathrm{G}$ & {$[\Omega \cdot \Omega]$} & 30840 & 37970 \\
\hline $\mathrm{k} \perp\left(\sigma_{\mathrm{z}}=1 \mathrm{~mm}\right)$ & {$\left[\mathrm{V} /\left(\mathrm{pC} \cdot \mathrm{cm}{ }^{2}\right)\right]$} & 0.23 & 0.38 \\
\hline $\mathrm{k} \|\left(\sigma_{\mathrm{z}}=1 \mathrm{~mm}\right)$ & {$[\mathrm{V} / \mathrm{pC}]$} & 1.46 & 1.72 \\
\hline
\end{tabular}

walls which make the chemical treatment and cleaning process extremely challenging but not impossible. This was proven very recently at Jlab on the $2.3 \mathrm{GHz} \mathrm{LL}$ single-cell cavity made of single crystal, demonstrating no field emission at $\mathrm{E}_{\mathrm{acc}}=45 \mathrm{MV} / \mathrm{m}\left(\mathrm{E}_{\text {peak }}=93 \mathrm{MV} / \mathrm{m}\right)$ and having very high intrinsic $\mathrm{Q}$, which, when scaled to $1.3 \mathrm{GHz}$ by factor $(2.3 / 1.3)^{2}$, would be $2 \cdot 10^{10}$ [5].

The dipole loss factor $\mathrm{k} \perp$ computed for bunch length $\sigma_{\mathrm{z}}=1 \mathrm{~mm}$ increased by $65 \%$ over the TESLA design which can be compensated by better cavity alignment in the linac. Beam dynamics studies are required to assess how severe an effect this change has on the beam emittance. The monopole loss factor $\mathrm{k} \|$ increased moderately by $18 \%$ and should not have any significant consequences on the beam quality.

\section{Multipacting and Lorentz Force Detuning}

The new cell geometry has been checked with respect to the multipacting phenomenon. No multipacting was found up to $\mathrm{E}_{\mathrm{acc}}=43 \mathrm{MV} / \mathrm{m}\left(\mathrm{E}_{\text {peak }}=100 \mathrm{MV} / \mathrm{m}\right)$. The Lorentz force detuning factor is not very different for the new shape from its value for the TESLA shape. For both shapes with optimally positioned stiffening rings the detuning at $45 \mathrm{MV} / \mathrm{m}$ is $1.49 \mathrm{kHz}$ and $-1.64 \mathrm{kHz}$ for the TESLA and LL-shape respectively. In both cases it was assumed that the wall thickness is $2.8 \mathrm{~mm}$. The computer simulation done at FNAL showed that an additional 10\% of wall thickness will reduce the detuning of the LL shape to the TESLA level.

\section{END-CELL GEOMETRY}

The end-cell geometry being very crucial for HOM damping is still under investigation. There are at least two approaches to optimize their geometry for optimal HOM damping. The difference between them arises from the damping of the 3-rd dipole passband which has two modes with high $(\mathrm{R} / \mathrm{Q})$. The first approach adopted by $\mathrm{KEK}$, is to keep these modes propagating in an interconnection between two adjacent cavities. In the case of long linear accelerators the interconnections should be

\footnotetext{
*With optimally located stiffening ring: TESLA shape at $r=54 \mathrm{~mm}$, LL-shape at $r=44 \mathrm{~mm}$ when the wall thickness is $2.8 \mathrm{~mm}$.
}

possibly kept short to avoid unnecessary lengthening of the machine. This space is very limited and an additional broadband absorber between cavities is also technically difficult. Therefore one would rather prefer that the damping will be provided by the HOM couplers instead. Mode propagation in the interconnection may also lead to unfortunate standing waves localized between cavities since notches and crests locations depend on frequency differences of the adjacent structures. Generally speaking, fabrication errors on the cavities will spread the HOM frequencies such that the situation is to some extend random. This has been observed for the 3-rd passband in some TTF cavities. As a result, a few cavities showed an order of magnitude less damping than expected for the highest $(\mathrm{R} / \mathrm{Q})$ mode from this passband because of the standing wave effect.

The second approach would require a longer effort but is important for the final version of ILC LL-cavity. It may not necessarily meet the "proof of principle" test which is the demonstration of very high gradients (>45 MV/m) and high intrinsic Qs which is the goal of the first approach. The second approach (SLAC, DESY, JLab) focuses on the development of an end-cell geometry that will not only guarantee suppression of HOMs up to 3-rd passband, but also damp the 5-th dipole passband as well. To achieve this will require extensive $2 \mathrm{D}$ and $3 \mathrm{D}$ computer modeling. The various end-cells under consideration are shown in Fig. 2. Their FM parameters are listed in Table 2 together with their beam-pipe diameters. The listed data confirm
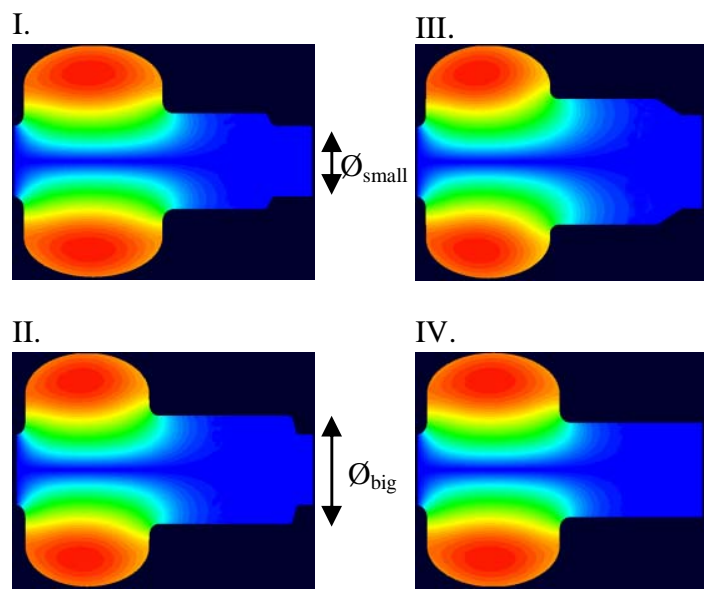

Figure 2: End-cells: I. DESY/SLAC first version; II. DESY/SLAC improved version; III. and IV. KEK versions. Pictures illustrate $\mathrm{H}$ field contour of FM.

Table 2: Parameters of end-cells.

\begin{tabular}{|l|c|c|c|c|c|}
\hline Parameter & Unit & I & II & III & IV \\
\hline$\emptyset_{\text {big }}$ & {$[\mathrm{mm}]$} & 82 & $\mathbf{9 2}$ & $\mathbf{1 0 8}$ & $\mathbf{8 0}$ \\
\hline$\emptyset_{\text {small }}$ & {$[\mathrm{mm}]$} & 60 & 60 & 80 & 80 \\
\hline $\mathrm{R} / \mathrm{Q}$ & {$[\Omega]$} & 123.3 & 115.5 & 103.1 & 124.7 \\
\hline $\mathrm{G}$ & {$[\Omega]$} & 288.4 & 291.5 & 297.5 & 287.6 \\
\hline $\mathrm{R} / \mathrm{Q} \cdot \mathrm{G}$ & {$[\Omega \cdot \Omega]$} & 35560 & 33668 & 30672 & 35864 \\
\hline
\end{tabular}


that opening up the beam pipe strongly reduces the $(\mathrm{R} / \mathrm{Q}) \cdot \mathrm{G}$ product of an end-cell and it should be done to a limited extent in seeking good damping of the HOMs.

\section{9-CELL STRUCTURE MODELING}

Following the first approach, KEK optimized the endcells for the 9-cell structure in 2D while SLAC/ FNAL/DESY pursued the second approach using 2D and 3D modeling $[6,7]$ on end-cells of type I. Table 3 shows the 3D modeling data for the 9-cell structure with endcells of type-I. The structure consists of a FM coupler and two HOM couplers identical to those in the TESLA structures (Fig. 3). Accelerating mode and HOMs with significant $(\mathrm{R} / \mathrm{Q})$ are listed in Table 3. Also data for two modes from the 5-th dipole passband is displayed since these have a tendency to be trapped. Both polarizations of the first mode from the 3-rd passband are insufficiently damped in the type-I end-cells. Work on the improved type-II version is in progress and 2D optimization this far has led to increased stored energy in the end-cell for this mode almost by a factor of 2 . One expects that the $\mathrm{Q}_{\mathrm{ext}}$ for this mode will be lowered by the same factor and be within specification $\left(\mathrm{Q}_{\mathrm{ext}}<10^{5}\right)$. For the 5-th passband the type-II end-cell has less stored energy by a factor $\sim 2.5$ than type-I, but the impedance of these modes would be still within specification $\left(<1.5 \mathrm{M} \Omega / \mathrm{cm}^{2}\right)$. If 3D modeling confirms these results the next step would be to test for their sensitivity to HOM frequency errors.

Table 3: FM and HOM data.

\begin{tabular}{|l|c|c|c|}
\hline \multicolumn{1}{|c|}{ Mode } & $\begin{array}{c}\mathbf{f} \\
{[\mathbf{M H z}]}\end{array}$ & $\begin{array}{c}(\mathbf{R} / \mathbf{Q})^{*} \\
{\left[\mathbf{\Omega} / \mathbf{c m}^{\mathbf{n}}\right]}\end{array}$ & $\mathbf{Q}_{\text {ext }}$ \\
\hline M: TM010-9 & 1300.00 & 1161 & $8 \cdot 10^{5}$ \\
\hline D: TE111-7a & 1717.15 & 5.0 & $4 \cdot 10^{4}$ \\
\hline D: TE111-7b & 1717.21 & 5.0 & $5 \cdot 10^{4}$ \\
\hline D: TE111-8a & 1738.12 & 3.0 & $6 \cdot 10^{4}$ \\
\hline D: TE111-8b & 1738.15 & 3.0 & $8 \cdot 10^{4}$ \\
\hline D: TM110-2a & 1882.15 & 3.4 & $6 \cdot 10^{3}$ \\
\hline D: TM110-2b & 1882.47 & 3.4 & $6 \cdot 10^{3}$ \\
\hline D: TM110-4a & 1912.04 & 4.6 & $9 \cdot 10^{3}$ \\
\hline D: TM110-4b & 1912.21 & 4.6 & $1 \cdot 10^{4}$ \\
\hline D: TM110-5a & 1927.10 & 15.6 & $1.5 \cdot 10^{4}$ \\
\hline D: TM110-5b & 1927.16 & 15.6 & $1.5 \cdot 10^{4}$ \\
\hline D: TM110-6a & 1940.25 & 12.1 & $2 \cdot 10^{4}$ \\
\hline D: TM110-6b & 1940.27 & 12.1 & $2 \cdot 10^{4}$ \\
\hline M: TM011-6 & 2177.48 & 192 & $10^{4}$ \\
\hline M: TM011-7 & 2182.81 & 199 & $10^{4}$ \\
\hline D: 3-rd-1a & 2451.07 & 31.6 & $1 \cdot 10^{5}$ \\
\hline D: 3-rd -1b & 2451.15 & 31.6 & $2 \cdot 10^{5}$ \\
\hline D: 3-rd 1-2a & 2457.04 & 22.2 & $5 \cdot 10^{4}$ \\
\hline D: 3-rd 1-2b & 2457.09 & 22.2 & $5 \cdot 10^{4}$ \\
\hline D: 5-th - 7a & 3057.43 & 0.5 & $3 \cdot 10^{5}$ \\
\hline D: 5-th - 7b & 3057.45 & 0.5 & $3 \cdot 10^{5}$ \\
\hline D: 5-th - 8a & 3060.83 & 0.4 & $8 \cdot 10^{5}$ \\
\hline D: 5-th - 8b & 3060.88 & 0.4 & $9 \cdot 10^{5}$ \\
\hline D-dipole; a, b indicate polarizations. & & \\
\hline n 0 for monopoles, n 32 for dipoles; M-monopole, & \\
\hline
\end{tabular}

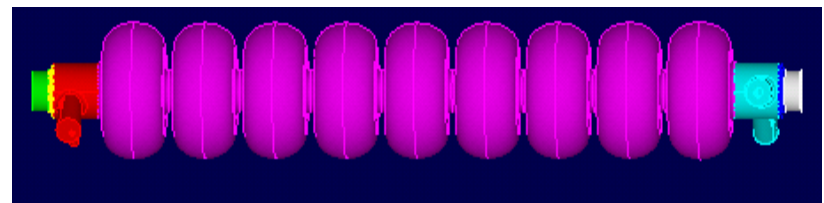

Figure 3. ILC 9-cell LL structure with end-cells type-I.

\section{FINAL REMARKS AND FUTURE PLANS}

We presented the LL-cavity design as a possible alternative to the TESLA baseline cavity design. To accept it as a better option we need to demonstrate all its potential advantages of higher gradient and higher intrinsic Q. Towards this goal KEK has embarked on a very ambitious schedule to fabricate and test four of the LL cavities by the end of summer 2005. In parallel, there will also be an effort at Jlab to develop a 9-cell aluminum model of the ILC LL structure for HOM damping verification. Then, the 7-cell single crystal $\mathrm{Nb}$ prototype will be built to demonstrate performance of the LL shape using the superior superconducting material and with a second infrastructure besides KEK for the superconducting surface preparation (buffered chemical polishing vs. EP ).

If the performance of the fundamental mode for the LL cavity shape turns out to be as expected, then work on optimizing the interconnection between cavities will proceed forward. This will comprise a large computational effort in which an entire chain of eight to twelve cavities will be modeled under the realistic conditions that the dipole frequencies will be randomly distributed because of loose fabrication tolerances.

\section{REFERENCES}

[1] E. Haebel et al., "Cavity Shape Optimization for a Superconducting Linear Collider", HEACC, Vol. 2, Hamburg 1992.

[2] J. Sekutowicz et al., "Low Loss Cavity for the $12 \mathrm{GeV}$ CEBAF Upgrade“, JLAB, TN-02-023, June 2002.

[3] K. Saito, "Fundamental RF critical Field Overview", Proceed. Of Workshop on Pushing the Limits of RF Superconductivity, ANL, Argonne, September 2004.

[4] J. Sekutowicz, "New Geometry Overview", Proceed. Of Workshop on Pushing the Limits of RF Superconductivity, ANL, Argonne, September 2004.

[5] P. Kneisel et al., "Preliminary Results from Single Crystal and Very Large Crystal Niobium Cavities", these Proceedings.

[6] Z. Li et al., "X-band Linear Collider R\&D in Accelerating Structures Through Advanced Computing", Proceed. of the 9th European Particle Accelerator Conference, 2004.

[7] L. Lee et al., "Modelling RF Cavity with External Coupling", 2005 SIAM Conference on Computational Science and Engineering, Orlando, Florida, 2005. 